\title{
La especificidad del contexto indígena como requerimiento para la formación inicial del profesorado*
}

\author{
Indigenous context specificity as teacher elementary education requirement \\ A especificidade do contexto indígena como requerimento para a formação inicial do \\ professorado
}

\author{
Omar Turra, ${ }^{\text {a }}$ Donatila Ferrada, ${ }^{\mathrm{b}}$ Alicia Villena ${ }^{\mathrm{c}}$ \\ ${ }^{a}$ Universidad Católica de Temuco, Facultad de Educación. Telf.: 45-2553824. \\ Correo electrónico: oturra@uct.cl \\ bUniversidad Católica de la Santísima Concepción, Facultad de Educación. Telf.: 41-2345393. \\ Correo electrónico: dferrada@ucsc.cl \\ ${ }^{b}$ Universidad Católica de la Santísima Concepción, Facultad de Educación. Telf.: 41-2345367. \\ Correo electrónico: avillena@ucsc.cl
}

\begin{abstract}
RESUMEN
Este artículo tiene como propósito central identificar requerimientos específicos de formación inicial de profesores para contextos de indigeneidad, desde las propias voces y experiencias del profesorado en ejercicio laboral en escuelas ubicadas en contextos de alta vulnerabilidad urbana y rural. Se trabajó desde una metodología comunicativa crítica con un diseño cualitativo, construyéndose 4 casos territorializados en la región del Bío-Bío. Para la recolección de la información se usó la técnica de grupo de discusión comunicativo y para su análisis la distinción de dimensiones transformadoras y exclusoras. Los resultados definen requerimientos de formación inicial en dos ámbitos: praxis curricular y desempeño en indigeneidad, los que posicionan la especificidad de este contexto como eje a considerar en los procesos de renovación curricular.
\end{abstract}

Palabras clave: formación inicial, renovación curricular, contexto de indigeneidad, vulnerabilidad educativa.

\begin{abstract}
This paper identifies core teacher requirements to teach high-need and culturally-diverse students of indigenous background. Using a Communicative Critical Methodology, four public and suburban Southern Chile elementary schools were studied on the basis of communicative discussion groups, questionnaires and group interviews. Data collected show both transforming and still tied to traditional approaches to teach socially vulnerable and culturally diverse students of indigenous background.
\end{abstract}

Key words: elementary teaching, curricular renovation, indigenous contexts, education vulnerability.

\section{RESUMO}

Este artigo tem como propósito central identificar requerimentos específicos de formação inicial de professores para contextos de indigeneidade, desde as próprias vozes e experiências do professorado em exercício laboral nas escolas localizadas em contextos de alta vulnerabilidade urbana e rural. Trabalhou-se desde uma metodologia comunicativa crítica com um desenho qualitativo, construindo-se 4 casos territorializados na região do Bío-Bío. Para a recolecção de informação, utilizou-se a técnica do grupo de discussão comunicativo, e para a análise utilizou-se a distinção de dimensões transformadoras e excluidoras. Os resultados definem requerimentos de formação inicial em dois âmbitos; praxe curricular e desempenho em indigeneidade, que posicionam a especificidade do contexto como um eixo a considerar nos processos de renovação curricular.

Palavras chave: formação inicial, renovação curricular, contexto de indigeneidade, vulnerabilidade educativa.

* Artículo elaborado en el marco del Proyecto Fondecyt-Conicyt $\mathrm{N}^{\circ} 1110130$. "Requerimientos profesionales de formación inicial de profesores para un desempeño de calidad en contextos de vulnerabilidad social y económica". 


\section{INTRODUCCIÓN}

Las demandas por cambios educacionales que con gran fuerza se han hecho sentir en el país han visibilizado los escenarios múltiples, variados e inequitativos en los que se desarrollan los procesos formativos en el sistema escolar chileno. Todo indica que la escuela ya no puede ser reducida simbólicamente a una institución única y homogénea, sino que por el contrario su sello explicativo se asocia con la diversidad de los espacios, geografías y comunidades, es decir, con los contextos.

Una realidad educativa vinculada a lo diverso y específico es la que ocurre cotidianamente en contexto escolar indígena mapuche. Aquí se desarrollan procesos formativos en que conviven sentidos y prácticas culturales diferenciadas, propuestas educativas que recogen, con diversos énfasis, las orientaciones de la política pública sobre educación para pueblos indígenas y aquellas asociadas a la noción de vulnerabilidad social y económica. Desafortunadamente, esta especificidad también se ha expresado en la conformación de un espacio tradicional de incubación de mecanismos de violencia simbólica, discriminación y desigualdad en las oportunidades educativas (Bourdieu y Passeron, 1996; Rother, 2005; Quilaqueo, Merino y Saiz, 2007). Ilustra esta desigualdad la brecha en las tasas de analfabetismo existente entre el pueblo mapuche y la población no indígena en Chile, que alcanza un promedio de 5 puntos; en cuanto a años de estudio entre el rango de edad 16 a 29 años la brecha es de 1,3 años, y respecto del ingreso a estudios superiores sólo el 7,9\% de los estudiantes mapuche accede a este sistema en comparación al 16,8\% de los no indígenas, es decir, la brecha es de 9 puntos promedio, según estadísticas oficiales del último censo nacional (INE-MIDEPLAN, 2005).

No obstante, este trato discriminatorio que ha tenido el Estado chileno con el pueblo mapuche, se ha pretendido revertir -aunque con poco éxito- a partir de la promulgación de la Ley indígena 19.253 (1993), en cuyos título se encuentra regulado las demandas educativas específicas para revalorizar su cultura y lengua. De esta normativa, surgió el programa piloto de educación intercultural bilingüe (1996) el que se institucionalizó definidamente como Programa (PEIB) el año 2000 y cuyo objetivo central se orienta a contribuir a mejorar los logros de aprendizaje, a partir del fortalecimiento de la identidad étnica de los educandos indígenas de establecimientos educacionales de Educación Básica ubicados en contexto de diversidad cultural y lingüística. También, la reforma educativa nacional de fines de los noventa reconoce y considera la diversidad cultural en la formación del estudiantado, tanto a nivel de producción curricular como en los procesos pedagógicos implementados por el profesorado. Un nuevo avance en la materia constituye el Decreto Supremo de Educación No 280, recientemente dictado (2009), que introduce el sector de aprendizaje Lengua Indígena en áreas de alta población escolar indígena.

Sin embargo, la información de campo evidencia una distancia y tensión entre lo dispuesto por la política pública educativa en contexto indígena y las condiciones en que se han implementado estos programas de mejoramiento educativo, puesto que uno de los principales agentes educativos que debe articular las orientaciones y propósitos de estos programas, como es el profesorado, no ha recibido formación específica para el trabajo en indigeneidad. 


\section{PROBLEMATIZACIÓN}

Como es sabido, la renovación curricular en la formación inicial docente ha adquirido particular importancia en Chile en las últimas tres décadas. En este propósito, desde la política pública se han configurado distintos programas, en correspondencia con las exigencias de la reforma educativa y la adopción de referentes internacionales en la materia, para definir los nuevos saberes y competencias que debiese poseer el profesorado en el contexto de la sociedad del conocimiento. ${ }^{1}$

Estos programas de renovación curricular se fundamentan en una racionalidad estandarizadora de la formación inicial, de tal manera que ninguno de ellos contempla la dimensión de indigeneidad en sus objetivos, a pesar de las disposiciones legales de educación en contexto indígena que así lo demandan y aquellos comprometidos en los convenios internacionales de reciente ratificación. ${ }^{2}$

La política pública en educación para pueblos indígenas, desde el ámbito normativo, plantea el manifiesto propósito de preservar la cultura y la lengua de estos pueblos a través de transformaciones curriculares que incorporan elementos propios de la cultura indígena en los procesos formativos del sistema educacional, tanto a nivel escolar como en la educación superior. Sin embargo, estas definiciones de política pública no se han traducido en transformaciones en el currículum de formación inicial de profesores ${ }^{3}$ y menos aún en una política de formación en la enseñanza superior que contemple una educación para o desde los pueblos indígenas (Williamson, 2008). Los profesores que se desempeñan en contextos educativos indígenas entran en contacto con formas culturales y cosmovisiones diferenciadas, además de especificidades curriculares y pedagógicas, aspectos de los que no adquirieron conocimiento en su proceso de formación inicial. Por consiguiente, el profesorado en ejercicio en estos contextos asumen un desempeño laboral para el cual no ha sido preparado (Quintriqueo, 2010). Esto ha generado que los docentes deban asumir en forma personal los desafíos que le demanda este tipo de desempeño, situación que genera profundas tensiones en cuanto a su ejercicio profesional y los propósitos de las normativas educativas orientadas a estos contextos.

Por otra parte, las decisiones de política pública que han introducido modificaciones curriculares y pedagógicas en contexto escolar indígena, tampoco han considerado el saber acumulado en las experiencias de los propios docentes en ejercicio en estos contextos. Si bien la generalidad de estos profesores carece de una formación específica para

1 Los programas referenciados son: el Proyecto de Mejoramiento de Formación Inicial Docente (PFFID), que implicó un proceso de renovación curricular de las carreras de pedagogía a fin de ajustarlas al marco referencial de la reforma educativa; el Programa de Mejoramiento de la Calidad de la Educación Superior (MECESUP), actualmente en vigencia, que implica la renovación curricular de las carreras de pedagogía para ajustarla al modelo basado en competencias en el marco del proyecto Tunning; y el sistema de Acreditación Nacional de las carreras de pregrado (actual CNA), en cuyo contexto es obligatoria la acreditación para las carreras de pedagogía, también de acuerdo a un diseño curricular basado en competencias.

2 Convenio 169 de la OIT Sobre Pueblos Indígenas y Tribales Independientes, ratificado por Chile, el año 2008, en su Art. 27. Los programas y los servicios de educación destinados a los pueblos interesados deberán desarrollarse y aplicarse en cooperación con éstos a fin de responder a sus necesidades particulares, y deberán abarcar su historia, sus conocimientos y técnicas, sus sistemas de valores y todas sus demás aspiraciones sociales económicas y culturales.

3 Un programa excepcional al respecto es la Pedagogía en Educación Básica Intercultural Bilingüe en Contexto Mapunche de la Universidad Católica de Temuco. 
educación en indigeneidad, su trayectoria de desempeño en el medio los hace portadores de un conocimiento en su hacer educativo importante de relevar. Su saber profesional puede reportar interesantes datos al proceso de formación inicial docente, considerando que se trata de subjetividades territorializadas en el complejo cultural, a la vez que se constituyen en fuentes de información más abarcadoras (dimensiones sociales y culturales) de una realidad educativa.

En consideración a los antecedentes planteados es que esta investigación tiene como propósito central levantar demandas curriculares de formación inicial desde las voces y experiencias de los profesores en ejercicio en contextos de indigeneidad, a fin de reportar insumos para una propuesta de un currículum de formación inicial que considere el componente poblacional indígena y, particularmente, su dimensión cultural.

\section{MARCO TEÓRICO}

La formación inicial docente en América Latina y en Chile se ha constituido en un ámbito de intervención relevante de las políticas educativas que buscan mejorar la calidad de los aprendizajes. Resulta un entendimiento común, que al momento de pensar en cualquier transformación pedagógica deba considerarse el rol preponderante del docente en el proceso educativo y, consecuentemente con ello, en la calidad de su formación. Esta comprensión está en línea con la literatura que posesiona a la actuación docente como elemento clave de cualquier transformación educativa (Fullan, 2002; Aguerrondo, 2004; Vaillant, 2005).

No obstante el discurso que indica al profesorado como actores fundamentales del cambio educativo y las políticas de renovación curricular y fortalecimiento profesional de los docentes, los programas de formación inicial presentan una escasa reflexión respecto del sentido de la enseñanza en los actuales escenarios de escolaridad y una insuficiente preparación para trabajar en contextos socioculturales diversos (Vezub, 2007). En esta perspectiva, se encuentran estudios que describen el desencuentro entre la formación inicial recibida y la realidad profesional presente en la diversidad de contextos escolares de los sistemas educativos (Bustos, 2007; González-Vallinas, Oterino y San Fabián, 2007).

En Chile la formación inicial se organiza en base a un desempeño homogéneo, es decir, prepara para atender pedagógicamente a un estudiante promedio y perteneciente a la cultura occidental. Con ello se unifica y estandariza la diversidad de desempeños a los cuales podría enfrentarse el futuro profesor, desconsiderando la especificidad de los territorios, cosmovisiones, de contextos culturales y la formación pedagógica en interculturalidad (Manzo y Westerhout, 2003: 10), aspecto que ha traído como consecuencia la profundización de la desigualdad de resultados de aprendizaje en sujetos e instituciones educativas.

Una excepción notoria al respecto constituye la Pedagogía en Educación Básica Intercultural dictada por la Universidad Católica de Temuco que tiene como propósito formar profesores interculturales a partir de la especificidad cultural mapuche para promover procesos formativos con especial consideración de la identidad cultural indígena (Quilaqueo, Quintriqueo y Cárdenas, 2005). Sin embargo, esta iniciativa es el resultado de acciones personales de equipos de académicos sensibilizados y comprometidos con el reconocimiento de esta cultura y no como la traducción de una política estatal al respecto. 
Por otra parte, para enfrentar los bajos resultados de aprendizaje, la política educativa ha categorizado las escuelas y sus estudiantes con indicadores de vulnerabilidad (IVE) que apuntan a favorecer con recursos económicos y programas de apoyo, los deficitarios resultados de aprendizaje escolar. Como consecuencia de esta política, las escuelas con alto componente escolar indígena en sus aulas adquieren la categoría de alta vulnerabilidad. No obstante, esta política no se vincula con procesos de formación inicial que aborden de forma especializada los espacios educativos caracterizados como vulnerables, afianzando con ello una práctica pedagógica homogénea.

La formación inicial homogeneizadora y la categorización en niveles de vulnerabilidad, desconocen la cultura de pertenencia de los sujetos escolares, lo que lleva a la escuela a instalar códigos descontextualizados que se mediatizan por un programa de estudios homogéneo (Pinto, 2008).

Como contrapartida a una educación homogeneizadora, la literatura científica en educación sugiere como uno de los pilares fundamentales para avanzar en la generación de resultados educativos igualitarios, la consideración de un currículum que incorpore el contexto cultural de los sujetos y sus comunidades que permitan articular iniciativas locales junto con condiciones prevalecientes en las escuelas (Harris y Chapman, 2004). Esto involucra avanzar hacia un entendimiento de la escuela vinculada con las comunidades sociales y los territorios en que se encuentran (Carrasco, 2008), donde los contenidos curriculares den cuenta de las formas culturales locales, que aporten a la autovaloración de la propia identidad, para desde ahí proyectarse en el conocimiento y valoración de las diferencias culturales en contextos más amplios (Pinto, 2008; Quintriqueo, 2010; Turra, 2012).

Una formación inicial pensada desde la consideración de los contextos culturales implica romper con la formación uniforme y avanzar hacia el diseño de currículos transformadores, que diversifiquen sus procesos de formación en función de la diversidad de contextos culturales (urbanidad marginal, ruralidad e indigeneidad) en que se desarrolle la acción educativa (Ferrada, Turra y Villena, 2013).

\section{METODOLOGÍA}

La metodología utilizada en la presente investigación es la comunicativa crítica que tiene como una de sus principales características, la ruptura epistemológica entre investigador e investigado y en que la construcción de significados se negocian desde la argumentación basada en criterios de validez (Gómez et al., 2006). En base a un diseño cualitativo se construyeron 4 casos caracterizados educativamente como de indigeneidad localizados en la provincia de Arauco (Región del Bío-Bío, Chile). Esta provincia es la de mayor concentración indígena mapuche en la región y una de las más altas del país $(12,7 \%)$; los aspectos cualitativos que la caracterizan resultan especialmente relevantes para sostener la dimensión de indigeneidad como ámbito de investigación en este contexto, a saber:

a. Históricamente, es el territorio tradicional de enfrentamiento con el conquistador español y, posteriormente, de resistencia frente al avance del Estado chileno.

b. Culturalmente, en este espacio se encuentran las comunidades tradicionales mapuche, ligadas a la ruralidad y a las costumbres ancestrales. 
c. Políticamente, en este territorio se ha desarrollado, en los últimos años, una parte importante del llamado "conflicto mapuche", centrado en las reivindicaciones de tierras que enfrentan a comuneros mapuche con el Estado chileno.

Desde este contexto, los casos se codificaron con las letras A, B, C y D y corresponden a escuelas básicas municipales (públicas) que imparten docencia de $1^{\circ}$ a $8^{\circ}$ año.

El caso A corresponde a una unidad educativa con un $20 \%$ de indigeneidad mapuche, el caso B con un $34 \%$, el caso C con $57 \%$ y el caso D con un $65 \%$, razón por la cual, estas dos últimas están incorporadas al programa intercultural bilingüe, no así las primeras.

Una caracterización específica de cada caso se describe a continuación:

Tabla 1. Caracterización

\begin{tabular}{|c|c|c|c|c|}
\hline Casos & Sector & $\begin{array}{c}\text { Tipo de contexto } \\
\text { demográfico y cultural }\end{array}$ & IVE del centro & $\begin{array}{c}\text { Matrícula del } \\
\text { centro }\end{array}$ \\
\hline A & Cañete & $\begin{array}{c}\text { Baja urbanidad-media } \\
\text { indigeneidad }\end{array}$ & 91 & 474 \\
\hline B & Lloncao & $\begin{array}{c}\text { Alta ruralidad-media } \\
\text { indigeneidad }\end{array}$ & 98 & 76 \\
\hline C & Los Huapes & $\begin{array}{c}\text { Alta ruralidad-alta } \\
\text { indigeneidad }\end{array}$ & 92 & 36 \\
\hline D & Paicaví & $\begin{array}{c}\text { Alta ruralidad-alta } \\
\text { indigeneidad }\end{array}$ & 82 & \\
\hline
\end{tabular}

Se trabajó con una muestra intencionada total de 23 profesores con un promedio de 24 años de desempeño laboral en el contexto.

La recogida de la información se realizó mediante la técnica de grupo de discusión comunicativo y se usó el análisis básico de la metodología comunicativa crítica que permite distinguir dimensiones transformadoras de exclusoras. Entendiendo las dimensiones exclusoras como aquellas barreras que algunas personas o colectivos encuentran y que les impiden incorporarse a una práctica o beneficio social, y las dimensiones transformadoras como aquellas que contribuyen a superar las barreras que impiden la incorporación de las personas y/o colectivos excluidos a prácticas o beneficios sociales (Gómez et al., 2006: 95-96).

\section{RESULTADOS}

\subsection{REQUERIMIENTOS CURRICULARES TRANSFORMADORES DE FORMACIÓN INICIAL}

Los hallazgos permitieron agrupar los requerimientos en dos dimensiones transformadoras, praxis curricular y desempeño laboral en indigeneidad. 
a. Requerimientos de praxis curricular en indigeneidad

Tabla 2. Requerimientos de praxis curricular

\begin{tabular}{|c|c|c|c|}
\hline Caso A & Caso B & Caso C & Caso D \\
\hline $\begin{array}{c}\text { No se } \\
\text { observa }\end{array}$ & $\begin{array}{c}\text { Formar para } \\
\text { trabajar } \\
\text { en cursos } \\
\text { multigrado y } \\
\text { con niños sin } \\
\text { preescolar. }\end{array}$ & $\begin{array}{c}\text { Formar para programar } \\
\text { el currículum en una } \\
\text { sala multigrado que } \\
\text { tiene requerimientos } \\
\text { específicos de } \\
\text { indigeneidad. }\end{array}$ & $\begin{array}{c}\text { Formar para programar } \\
\text { el currículum en una } \\
\text { sala multigrado con } \\
\text { indigeneidad. }\end{array}$ \\
\hline $\begin{array}{c}\text { No se } \\
\text { observa }\end{array}$ & No se observa & $\begin{array}{c}\text { Formar para programar } \\
\text { clases en conjunto } \\
\text { con la "educador/a } \\
\text { tradicional". }\end{array}$ & $\begin{array}{c}\text { Formar para programar } \\
\text { clases en conjunto con la } \\
\text { "educadora tradicional". }\end{array}$ \\
\hline $\begin{array}{c}\text { No se } \\
\text { observa }\end{array}$ & No se observa & $\begin{array}{c}\text { Formar en la cultura } \\
\text { y en la lengua } \\
\text { indígena para tratarla } \\
\text { curricularmente en aula. }\end{array}$ & $\begin{array}{c}\text { Formar en la cultura y en } \\
\text { la lengua indígena para } \\
\text { tratarla curricularmente } \\
\text { en aula. }\end{array}$ \\
\hline
\end{tabular}

Desde un análisis cultural de los casos, la dimensión praxis curricular en indigeneidad, emerge como un requerimiento de formación inicial en tres de los cuatro casos estudiados (B, C y D), donde los casos C y D son comunes a diferencia del caso B en que no se observa la demanda por el trabajo con el/la educador/a tradicional y la formación en la lengua y cultura indígena para el trabajo curricular en aula, aspecto que se corresponde con los primeros casos que están incorporados al programa de educación intercultural bilingüe, no así el último. Por su parte, el requerimiento saber trabajar con cursos multigrado se corresponde con las características de ruralidad que tienen los tres casos en los que se encuentra presente, por el contrario, no es demandado en el caso A dado que es una escuela urbana.

Desde un análisis demográfico, el caso urbano no presenta requerimientos de formación vinculados a un saber hacer curricular específico, sin embargo, los casos de ruralidad observan un requerimiento propio (programación de aula multigrado).

Por consiguiente, la dimensión praxis curricular en indigeneidad se define como un saber hacer curricular desde el contexto cultural y demográfico que tiene requerimientos específicos y diferenciados de otros contextos, aspecto que se ve claramente representado en lo cultural en el trabajo curricular con el/la educador/a tradicional, quien corresponde a una persona de la propia comunidad que tiene dominio y forma parte de la lengua y la cultura y que se suma al trabajo de aula junto al profesor/a responsable, y en lo demográfico, en la especificidad de la programación curricular de la ruralidad. 


\section{b. Requerimientos curriculares de desempeño en indigeneidad}

Tabla 3. Requerimientos de desempeño curricular

\begin{tabular}{|c|c|c|c|}
\hline Caso A & Caso B & Caso C & Caso D \\
\hline $\begin{array}{c}\text { Formar para } \\
\text { saber trabajar } \\
\text { pedagógicamente con } \\
\text { los apoderados y sus } \\
\text { familias en contexto de } \\
\text { indigeneidad. }\end{array}$ & $\begin{array}{c}\text { Formar para trabajar } \\
\text { con la familia como } \\
\text { apoyo pedagógico } \\
\text { para la escuela. }\end{array}$ & $\begin{array}{c}\text { Formar para } \\
\text { trabajar con } \\
\text { apoderados } \\
\text { y familias en } \\
\text { contexto de } \\
\text { indigeneidad. }\end{array}$ & $\begin{array}{c}\text { Formar para } \\
\text { trabajar con } \\
\text { apoderados } \\
\text { y familias en } \\
\text { contexto de } \\
\text { indigeneidad }\end{array}$ \\
\hline $\begin{array}{c}\text { Formar para establecer } \\
\text { redes de apoyo que le } \\
\text { permitan resolver la } \\
\text { diversidad de demandas } \\
\text { que tiene el contexto } \\
\text { indígena. }\end{array}$ & $\begin{array}{c}\text { Formar para trabajar } \\
\text { comunitariamente } \\
\text { para salir adelante y } \\
\text { ubicarse en igualdad } \\
\text { de condiciones con } \\
\text { la gran urbanidad. }\end{array}$ & No se observa. & $\begin{array}{c}\text { Formar para } \\
\text { trabajar }\end{array}$ \\
$\begin{array}{c}\text { Formar al futuro docente } \\
\text { en prácticas tempranas } \\
\text { e intensivas en función } \\
\text { de contextos de }\end{array}$ & $\begin{array}{c}\text { Profundizar las } \\
\text { prácticas progresivas } \\
\text { en el contexto } \\
\text { considerando la } \\
\text { vulnerabilidad indígena. }\end{array}$ & $\begin{array}{c}\text { Incorporar } \\
\text { prácticas más } \\
\text { tempranas y } \\
\text { más intensivas } \\
\text { en función de } \\
\text { contextos de }\end{array}$ & No se observa. \\
indigeneidad. & \\
\hline
\end{tabular}

Desde un análisis cultural, la dimensión desempeño en indigeneidad, se encuentra presente como requerimiento en todos los casos estudiados existiendo total coincidencia entre ellos, a excepción de la ausencia de uno de ellos en los casos $\mathrm{C}$ y $\mathrm{D}$, de forma independiente de si está incorporado o no al programa de educación intercultural bilingüe. Al mismo tiempo, se observa total coincidencia en los requerimientos demandados en los casos sin programa intercultural. Por su parte, desde un análisis demográfico, no se observa distinción.

Por consiguiente, la dimensión desempeño en indigeneidad se define como los requerimientos de formación vinculados directamente a las características culturales del contexto.

\subsection{REQUERIMIENTOS CURRICULARES EXCLUSORES DE DESEMPEÑO EN INDIGENEIDAD}

Los hallazgos permitieron construir una sola dimensión de carácter exclusor presente en dos de los casos estudiados: 
Tabla 4. Requerimientos exclusores

\begin{tabular}{|c|c|c|c|}
\hline Caso A & Caso B & Caso C & Caso D \\
\hline No se observa & & $\begin{array}{c}\text { Formar para enfrentar las } \\
\text { situaciones conflictivas } \\
\text { de las familias del } \\
\text { estudiantado indígena. }\end{array}$ & No se observa \\
\hline & $\begin{array}{c}\text { Formar al profesorado } \\
\text { sin considerar la cultura } \\
\text { y la lengua de la } \\
\text { comunidad indígena. }\end{array}$ & $\begin{array}{c}\text { Formar al profesorado sin } \\
\text { considerar la cultura y la } \\
\text { lengua de la comunidad } \\
\text { indígena. }\end{array}$ & \\
\hline
\end{tabular}

Desde un análisis cultural, llama la atención que los dos requerimientos exclusores encontrados están presentes en uno de los casos (C) con el programa de educación intercultural bilingüe, no así en el otro (D). Misma situación ocurre con el requerimiento presente en el caso sin programa de educación intercultural bilingüe (B respecto de A). Por su parte, desde un análisis demográfico, los requerimientos observados no están asociados a contenidos de esta naturaleza, dado su carácter eminentemente cultural.

Por consiguiente, la dimensión de desempeño en indigeneidad exclusora se define como un trabajo que deslegitima y omite el contexto cultural que atiende.

\section{DISCUSIÓN}

En términos generales, los hallazgos evidencian una fuerte presencia de la dimensión transformadora y una débil expresión de ellas en la dimensión exclusora. Este dato resulta muy significativo puesto que pone en evidencia que las demandas de los profesores se orientan a la apertura de espacios movilizadores de los procesos pedagógicos en contexto específico de indigeneidad.

En términos de contenidos, los resultados muestran dos grandes requerimientos: uno, la formación en praxis curricular de indigeneidad y el otro, la formación para desempeño en el contexto, que se distinguen porque el primero se presenta asociado al contexto cultural y demográfico, sin embargo, el segundo lo hace solo respecto del cultural tanto en su dimensión transformadora como exclusora.

El requerimiento de formación de praxis curricular en indigeneidad podría encontrar explicación en la necesidad de saber programar el currículum escolar considerando la especificidad cultural (indígena) en directa relación con las demandas del PEIB y la demográfica asociada exclusivamente a la ruralidad que tiene condicionantes propias de la programación curricular. Por otra parte, también podría establecerse un vínculo entre el concepto de praxis que supone un dinamismo permanente entre teoría y acción con el propio pensamiento indígena que es una relación entre el actuar y el saber, donde nada está aislado, nada está de más. Se trata de una visión integradora, holística y cíclica de la interacción entre las cosas (Álvarez, 2005) que pudiera estar siendo significado de alguna forma en este requerimiento transformador del profesorado.

Por su parte, el requerimiento de formación de desempeño en indigeneidad asociado solo al contexto cultural podría dar cuenta de las propias demandas de la comunidad 
indígena por la representación de su cultura en el currículum escolar que se expresaría a través del profesorado. Este hecho, emerge por sobre la definición de la política que implementa el programa intercultural bilingüe, lo cual permitiría explicar este requerimiento transformador del profesorado. Por el contrario, el requerimiento exclusor se corresponde con la visión discriminadora que portaría el profesorado respecto de este tipo de cultura y que perdura como un significado que aflora a la hora de caracterizarla.

En consecuencia, estos requerimientos construidos desde las voces del profesorado en ejercicio en contextos de indigeneidad con vulnerabilidad urbana y rural, interpelan los procesos de renovación de formación inicial actualmente en desarrollo, en la medida que éstos tienden a la homogeneización del currículum a nivel nacional. Particularmente preocupante es el desconocimiento de la normativa jurídica en el ámbito educativo para el contexto indígena (Decreto 280 y Convenio 169) que pretende dar cuenta de esta especificidad y que estos procesos de renovación curricular omiten.

También estos requerimientos se complementan con el planteamiento de la literatura que viene relevando la pertinencia cultural de los procesos pedagógicos (Torres, 1998; Besalú, 2002) con el propósito de valorizar y reforzar las identidades locales, abriéndose al mismo tiempo al diálogo con otras culturas. Esto involucra avanzar hacia un entendimiento de la escuela vinculada con las comunidades sociales y los territorios en que se encuentra en donde los contenidos curriculares den cuenta de las formas culturales locales, que aporten a la autovaloración de la propia identidad, para desde ahí proyectarse en el conocimiento y valoración de las diferencias culturales en contextos más amplios (Pinto, 2008; Quintriqueo, 2010).

\section{CONCLUSIONES}

El profesorado en ejercicio laboral en contextos de indigeneidad con alta vulnerabilidad rural y urbana realiza demandas curriculares de formación inicial que tiene que ver con competencias en praxis curricular vinculada al contexto cultural y demográfico, y con el desempeño laboral directamente vinculado al contexto cultural.

Las demandas curriculares transformadoras aportan saberes y competencias que desbordan los ámbitos de dominio del ejercicio docente respecto de la mantención de la formación inicial tradicional existentes en las instituciones de educación superior. Al mismo tiempo que posiciona la cultura indígena como parte constituyente del currículum de formación inicial. En cambio, la demanda de formación inicial exclusora se relaciona con el estereotipo occidental respecto de la indigeneidad.

Estos requerimientos resultantes de la investigación permiten recuperar la incorporación y consideración de los sujetos, los contextos y sus comunidades, tradicionalmente ausentes, en los procesos de formación inicial.

\section{REFERENCIAS BIBLIOGRÁFICAS}

Aguerrondo, I. (2004). Los desafíos de la política educativa relativos a las reformas de la formación docente. En AAVV., Maestros en América latina: Nuevas perspectivas sobre su formación y desempeño (pp. 97-142). Santiago de Chile: PREAL - CINDE. 
Álvarez, J. (2005). Introducción al contexto indígena. En: MINEDUC, Orientaciones para la contextualización de planes y programas para la educación intercultural bilingüe (pp. 15-18). Santiago: MINEDUC.

Besalú, X. (2002). Diversidad cultural y educación. Madrid: Síntesis.

Bourdieu, P. y Passeron, J. C. (1996). La reproducción: elementos para una teoría del sistema de enseñanza. México D.F: Fontamara.

Bustos, A. (2007). Enseñar en la escuela rural aprendiendo a hacerlo. Evolución de la identidad profesional en las aulas multigrado. Profesorado: Revista de Currículum y Formación del Profesorado, vol. 11, $n$ 3. Web: http://www.ugr.es/ recfpro/rev113COL5.pdf (10/12/2011).

Carrasco, A. (2008) Investigación en Efectividad y Mejora Escolar: ¿Nueva agenda? REICE, vol. 6, n. 4, 5-23.

INE-MIDEPLAN (2005). Estadísticas Sociales de los pueblos indígenas en Chile Censo 2002. Santiago: MAVAL.

Ferrada, D., Turra, O. y Villena, A. (2013). Currículum transformador de formación inicial para profesores en contextos de vulnerabilidad social. Cadernos de Pesquisa, vol. 43, n.149, 642-661.

Fullan, M. (2002). Las fuerzas del cambio. Explorando las profundidades de la reforma educativa. Madrid: Akal.

González-Vallinas, P., Oterino, D. y San Fabián, J. (2007). Factores asociados a la formación permanente del profesorado de educación secundaria en Asturias. Profesorado: Revista de Currículum y Formación del Profesorado, vol. 11, $n$ 1. Web: http://www.ugr.es/ recfpro/ rev111ART1.pdf (visitado el 12/12/2011).

Gómez, J., Latorre, A., Sánchez, M. y Flecha, R. (2006). Metodología comunicativa crítica. Barcelona: El Roure.

Harris, A. \& Chapman, C. (2004) Towards differentiated improvement for schools in challenging circumstances. British Journal of Educational Studies, vol. 54, n. 4, 417-431.

LEY INDÍGENA No 19.253 (1993). Protección, Fomento y Desarrollo de los Indígenas, y crea la Corporación Nacional de Desarrollo Indígena. Santiago: Biblioteca del Congreso Nacional de Chile.

Manzo, L. y Westerhout, C. (2003) Propuesta Metodológica en Educación Intercultural para contextos urbanos. Cuadernos Interculturales, vol. 1, n. 1, 3-47.

Pinto, R. (2008). El currículo crítico. Una pedagogía transformativa para la educación latinoamericana. Santiago: Ediciones PUC.

Quilaqueo, D., Quintriqueo, S. y Cárdenas, P. (2005). Educación, Currículum e Interculturalidad. Elementos sobre formación de profesores en contexto mapuche. Temuco: UC Temuco.

Quilaqueo, D., Merino, M. y Saiz, J. (2007). Representación social mapuche e imaginario social no mapuche de la discriminación percibida. Atenea, n. 496, 81-103.

Quintriqueo, S. (2010). Implicancias de un modelo curricular monocultural en contexto mapuche. Temuco: Universidad Católica de Temuco.

Rother, T. (2005). Conflicto intercultural y educación en Chile: Desafíos y problemas de la educación intercultural bilingüe (EIB) para el pueblo mapuche. Revista Austral de Ciencias Sociales, $n$. 9, 71-84.

Torres, J. (1998). Globalización e Interdisciplinariedad. El currículum integrado. Madrid: Morata.

Turra, O. (2012). Currículo y construcción de identidad en contextos indígenas chilenos. Educación y educadores, vol. 15, n. 1, 81-95.

Vaillant, D. (2005). Formación de docentes en América Latina. Re-inventando el modelo tradicional. Barcelona: Octaedro.

Vezub, L. (2007). La formación y el desarrollo profesional docente frente a los nuevos desafíos de la escolaridad. Profesorado. Revista de Currículum y Formación de Profesorado, vol. 11, $n$ 1. Web: http://www.ugr.es/ recfpro/rev111ART2.pdf (visitado el 13/12/2011).

Williamson, G. (2008) Educación universitaria y educación intercultural en Chile. Cuadernos Interculturales, vol. $6, n$. 10, 125-156. 
